\title{
Retraction
}

\section{Retracted: Design of a Narrow Bandwidth Bandpass Filter Using Compact Spiral Resonator with Chirality}

\author{
Active and Passive Electronic Components \\ Received 12 May 2019; Accepted 12 May 2019; Published 9 June 2019 \\ Copyright (C) 2019 Active and Passive Electronic Components. This is an open access article distributed under the Creative \\ Commons Attribution License, which permits unrestricted use, distribution, and reproduction in any medium, provided the \\ original work is properly cited.
}

Active and Passive Electronic Components has retracted the article titled "Design of a Narrow Bandwidth Bandpass Filter Using Compact Spiral Resonator with Chirality" [1]. The article took the figures, the table, and most of the text from an unpublished article by Drs. Mohammad Reza Khawary, Seyed Mohammad Hashemi, Vahid Nayyeri, and Mohammad Soleimani previously submitted to IEEE Microwave and Wireless Components Letters in August 2015. The other journal confirmed the details of that earlier submission.

The first author does not agree with retraction and says their laboratory conducted this work, which was done by a former, unnamed student, and any similarity is a coincidence. We could not contact the other authors. The first author provided what they said were original simulation and measurement results, and said different software was used during filter design, simulation, optimization, and fabrication: CST for EM simulation, HFSS for resonant frequency extraction, ADS for circuit simulation, and AutoCAD for drawing the layout for fabrication; the filter was measured using R\&S vector network analyzer.

\section{References}

[1] W. Li, Z. Tang, and X. Cao, "Design of a narrow bandwidth bandpass filter using compact spiral resonator with chirality," Active and Passive Electronic Components, vol. 2016, Article ID 7074392, 6 pages, 2016. 


\title{
Design of a Narrow Bandwidth Bandpass Filter Using Compact Spiral Resonator with Chirality
}

\author{
Weiping Li, ${ }^{1,2}$ Zongxi Tang, ${ }^{1}$ and Xin Cao ${ }^{1}$ \\ ${ }^{1}$ School of Electronic Engineering, University of Electronic Science and Technology of China, No. 2006 Xiyuan Ave, \\ West Hi-Tech Zone, Chengdu 611731, China \\ ${ }^{2}$ School of Information Engineering, East China Jiaotong University, No. 88 Shuanggang Road, Nanchang 330013, China
}

Correspondence should be addressed to Weiping Li; lwp8277@126.com

Received 8 October 2016; Revised 17 November 2016; Accepted 23 November 2016

Academic Editor: Gerard Ghibaudo

Copyright (C) 2016 Weiping Li et al. This is an open access article distributed under the Creative Commons Attribution License, which permits unrestricted use, distribution, and reproduction in any medium, provided the original work is properly cited.

In this article, a compact narrow-bandpass filter with high selectivity and improved rejection level is presented. For miniaturization, a pair of double negative (DNG) cells consisting of quasi-planar chiral resonators are cascaded and electrically loaded to a microstrip transmission line; short ended stubs are introduced to expand upper rejection band. The structure is analyzed using equivalent circuit models and simulated based on EM simulation software. For validation, the proposed filter is fabricated and measured. The measured results are in good agreement with the simulated ones. By comparing to other filters in the references, it is shown that the proposed filter has the advantage of skirt selectivity and compact size, so it can be integrated more conveniently in modern wireless communication systems and microwave planar circuits.

\section{Introduction}

Design of very compact microwave devices compatible with printed circuit board and monolithic-microwave integratedcircuit fabrication technologies has gained great interest in the last decades. The split-ring resonators (SRRs) and their counterparts, complementary split-ring resonators (CSRRs), are key aspects that propose new design strategies to miniaturize planar microwave circuit [1]. It is demonstrated that, by combining two metal levels at both sides of a dielectric layer connected by vias with an appropriate topology, it is possible to design new resonators with a higher level of miniaturization [2]. These resonators which often exhibit chirality [3] can be used to synthesize left handed structures with neither severe degradation in the quality factor [2] nor causing electromagnetic compatibility (EMC) problems dealing with SRR and CSRR structures $[3,4]$.

Narrow-bandpass filters (NBPFs) with sharp selectivity and high rejection are increasingly demanded in modern microwave communications systems. In order to miniaturize such filters for circuit integration, many research works have been done. In [5], composite right/left-handed coplanar waveguide (CRLH-CPW) resonators are used to create a passband. But in general, the parasitic effects of CPW on the coupling gaps usually cannot be neglected. Therefore, the selectivity would be compromised. In [6], CSRRs have been loaded on the top side of the substrate, but a good deal of insertion loss of the passband is caused by the coupling and fringing capacitance brought by this structure. Also, unwanted spurious responses occur. In [7], multisection stepped-impedance resonators are cascaded to create a relative wide passband. However, the resonant mode cannot be easily controlled and the design process is comparatively complex. In [8], electromagnetic bandgap (EBG) is introduced on the ground plane of the filter. Better out-ofband rejection has been achieved and the design process can be more flexible. But in most cases, the loss of the in-band frequency response is inevitably increased by the rejection effect of EBG. Therefore, some performances must be balanced to make compromises. In [9], SRRs are coupled together to create a single passband. Since the structures of SRRs and CSRRs are quite the same, similar problems have 


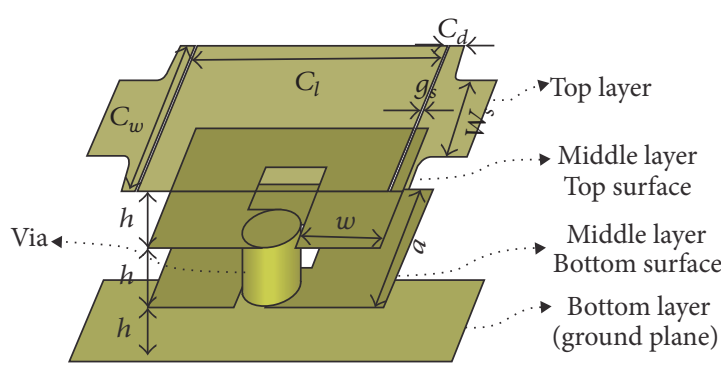

(a)

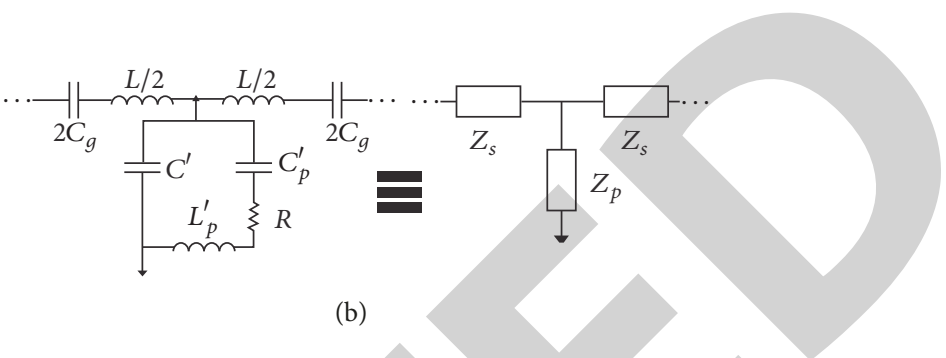

FIGURE 1: (a) 3D layout of the DNG cell including quasi-planar chiral resonator (particle) electrically loaded to a microstrip line with series gaps. (b) Lumped element equivalent circuit and equivalent T-model.

been encountered as that with CSRRs. Recently, other types of structures have been proposed to overcome the aforementioned disadvantages. In [10-17], composite right/lefthanded $(\mathrm{CRLH})$ metamaterial structure is demonstrated. CRLH resonators have positive, negative, and zeroth resonant modes based on the working condition. At zeroth resonant mode, the passband can achieve the highest selectivity with the lowest insertion loss due to the low conductor loss at this special resonant mode. In addition, CRLH resonators are often designed in the form of interdigital or spiral geometric structure, which makes the size more compact. In [1825], defected ground structure (DGS) has been proposed. DGSs are etched at the bottom plane of the resonators and band-stop frequency responses are achieved. Since DGS is independent of the resonators on the upper layer, the design process can be much more flexible. Out-of-band rejection can be increased by the slow-wave effect of DGS. Moreover, DGS are perfectly compatible with CRLH structures. In order to further miniaturize the size of the microwave circuit, in [26], multilayered quasi-planar structure is proposed. Different electromagnetic structures are packed into different layers of the substrate through weak coupling. Thus, more compact size has been achieved without major influence on the performances of the device. Inspired by these previous literatures, in our work, a novel NBPF with good selectivity and higher level of miniaturization using quasi-planar chiral resonators is proposed. The corresponding equivalent circuit has been analyzed and the results of full-wave simulation and experimental measurement have been presented.

\section{Filter Design}

Figure 1(a) shows the topology of the double negative (DNG) cell conceptually proposed in [4], consisting of a microstrip line with series gaps and a quasi-planar chiral resonator, electrically loaded to the transmission line. As illustrated in Figure 1(a), this topology can be printed on the surfaces of a three-layer substrate. The circuit model of the cell and its transformed T-model are depicted in Figure 1(b) (the circuit model is clearly described in [4]). In [4] it was demonstrated that this resonator provides negative permittivity in a narrow band after its resonant frequency. On the other hand, series gaps $\left(C_{g}\right)$ between transmission lines expose

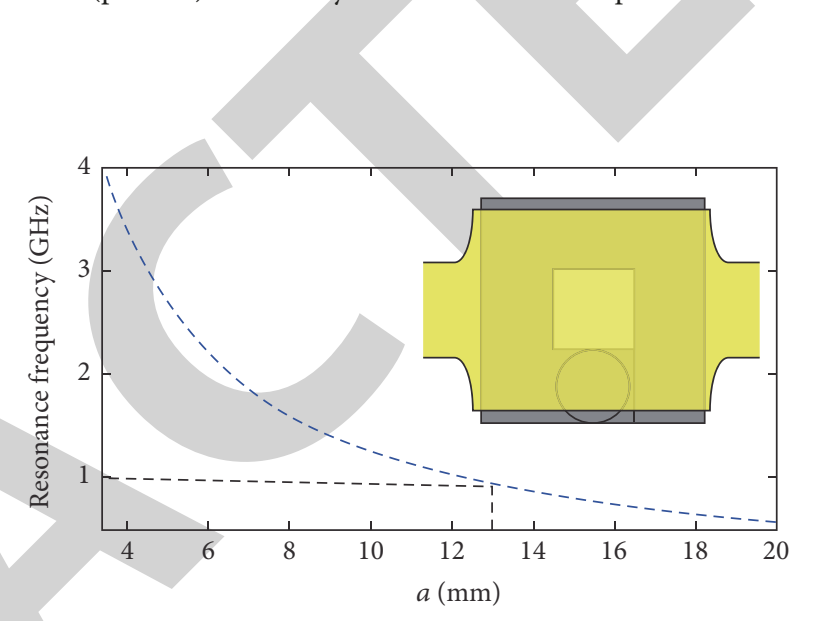

FIGURE 2: The variation of the resonant frequency of the chiral particle with respect to changing " $a$ " (depicted in Figure 1). Other dimension parameters are set to $w \approx a / 3, W_{s}=5.5 \mathrm{~mm}, C_{l}=10 \mathrm{~mm}$, $C_{w}=11.4 \mathrm{~mm}$, and $C_{d}=1.4 \mathrm{~mm}$.

negative permeability before their plasma frequency. Thus, this subwave-length structure with appropriate topology supports backward-wave propagation in a narrow frequency band. Therefore, the main idea behind this work is to use this DNG cell for implementing a compact NBPF.

Because the particle is excited with electrical field perpendicular to the substrate, for better coupling, the transmission line above the particle is widened. In addition, this widening prevents gap size from being closer to the limits imposed by the fabrication technology (approximately $0.1 \mathrm{~mm}$ ). The line is tapered next to the widened section for a better matching.

Particle resonant frequency is determined by its dimensions. Among the dimension parameters indicated in Figure 1(a), to the side length of the loop, " $a$ " is the most important one on the resonant frequency. Figure 2 shows the resonant frequency variation of the resonator with respect to changing " $a$." In the simulation, three layers of Rogers 4003C substrate with relative dielectric constant $\left(\varepsilon_{r}\right)$ of 3.55, thickness $(h)$ of $0.813 \mathrm{~mm}$, and loss tangent $\delta$ of 0.0027 are used and copper metallization thickness is $35 \mu \mathrm{m}$. To realize the passband at $1 \mathrm{GHz}$, the value of " $a$ " is set to be $12.9 \mathrm{~mm}$.

While setting the gap size $\left(g_{s}\right)$, it can be noticed that larger $g_{s}$ provides more suitable rejection up to the plasma frequency but results in a higher insertion loss within the 


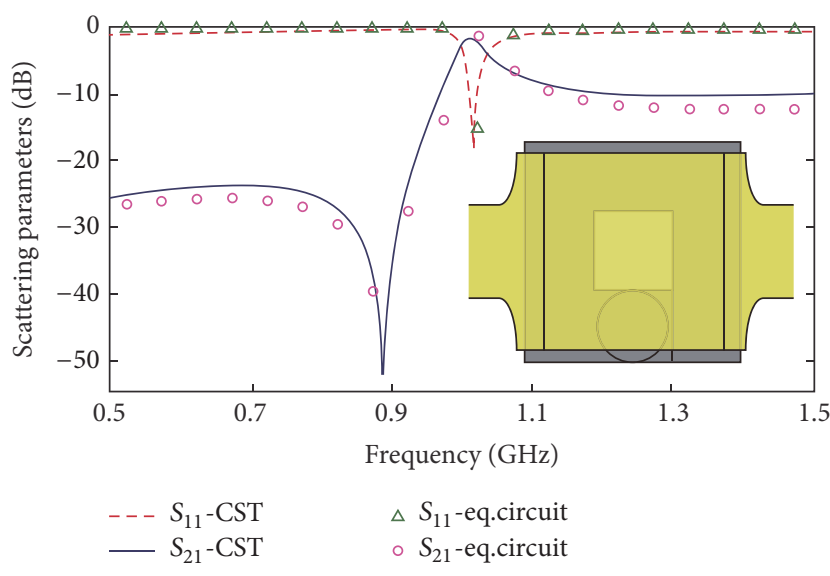

FIGURE 3: Scattering parameters of the DNG cell. The elements of the equivalent circuit are extracted as $C_{p}^{\prime}=4.396 \mathrm{pF}, L_{p}^{\prime}=7.308 \mathrm{nH}$, $R=0.6 \Omega, C^{\prime}=10.24 \mathrm{pF}, L=4.182 \mathrm{nH}$, and $C_{g}=0.8575 \mathrm{pF}$.

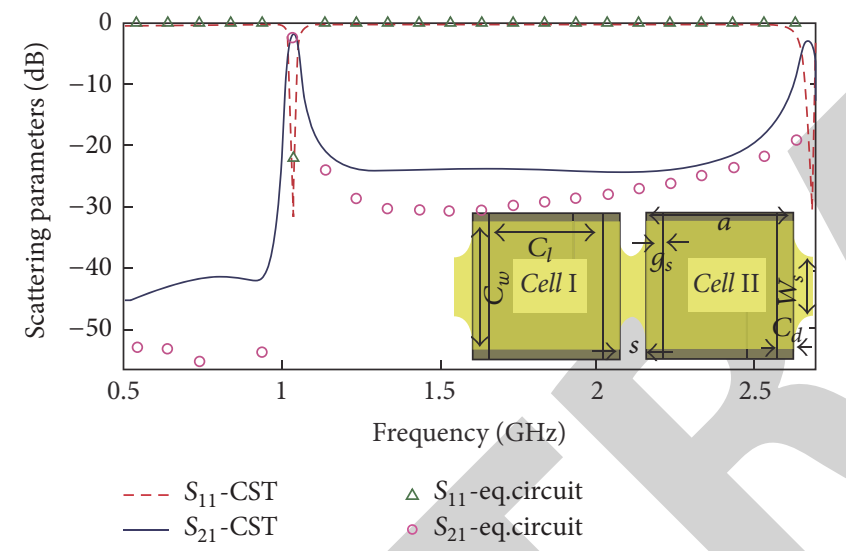

Figure 4: Scattering parameters of two cascade DNG cells $(s=$ $2.1 \mathrm{~mm})$.

passband. On the other hand, smaller $g_{s}$ decreases the rejection level within the stopband. In this design $g_{s}$ is set to be $0.2 \mathrm{~mm}$ to lessen the influence caused by fabrication uncertainties.

The results of full-wave electromagnetic and equivalent circuit simulations of the structure shown in Figure 1(a) are depicted in Figure 3, which clearly shows a narrowpassband around $1 \mathrm{GHz}$ (the equivalent circuit parameters of the topology are extracted from equations (14), (15), and (17)(19) of [4] and given in the caption).

To achieve a deeper rejection and also a sharper passband, two DNG cells are cascaded as shown in Figure 4, where cell I and cell II contain a left-handed and a righthanded chiral resonator, respectively. Figure 4 also shows the simulations results. Comparing to the results shown in Figure 3, improvement in filter performance is obvious. Also, the spurious response occurs far beyond twice the resonant frequency of the resonators, and the out-of-band rejection has been improved.

In addition, to expand upper rejection band and improve selectivity, a transmission zero is placed at $2.6 \mathrm{GHz}$ using

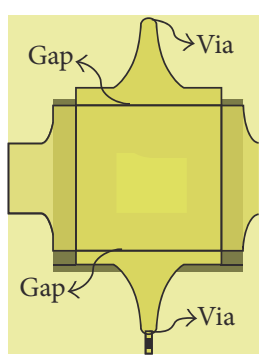

(a)

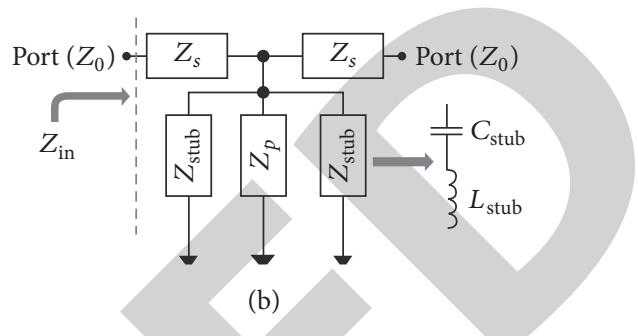

FIgURE 5: A DNG cell with loaded stubs; (a) layout and (b) equivalent circuit. short ended stubs coupled with gaps to the wide sections of the transmission line as shown in Figure 5(a). In Figure 5(b) the equivalent circuit of a cell loaded with short ended stubs is depicted where the stubs (with coupling gaps) are modeled by $L C$ resonators $\left(Z_{\text {stub }}\right)$. Appendix describes how to extract the elements of the equivalent circuit, that is, $L_{\text {stub }}$ and $C_{\text {stub }}$. These capacitors and inductors can achieve different resonant modes with the change of operating frequency.

\section{Experimental Results and Discussions}

Three layers of Rogers RO4003C are used to fabricate the proposed filter. The transmission line with gaps and stubs are printed on the top surface of the top layer. Two metal rings are printed on each surface of the middle layer and connected to each other using copper ribbons to form quasi-planar resonators. The bottom layer is ground plane (depicted in Figure 6). Then, the three layers are connected using insulating gel. After gluing, the structure is punctured and the vias of the stubs are soldered with tin. Then, by using the substrate with less thickness, stronger coupling between the upper and lower resonator rings can be obtained with the reducing of resonant frequency, which indicates that further miniaturization could be achieved. Moreover, advanced monolithic fabrication method can mitigate some problems due to the lack of complete alignment among the layers (especially between the first and the second layers) and the substrate loss of the gel.

The results obtained from equivalent circuit simulation, full-wave simulation, and measurements are shown in Figure 7, demonstrating good agreement between them; however, use of copper ribbons instead of vias has effect on the resonant frequency of the resonators which appears by a slight frequency-shift in the measurement results. The small differences between simulated and measured results are mainly due to fabrication inaccuracy. But in general, the measured results are in good agreement with the simulated results.

Finally the proposed filter is compared with the other compact NBPFs reported in the literature in terms of resonator type, center frequency $\left(f_{0}\right)$, fractional bandwidth (FBW), length $\left(\lambda_{g}\right)$, and insertion loss (IL), where $\lambda_{g}$ is the guided wavelength of the electromagnetic waves in the substrate at the resonant frequency. The comparison is shown 
TABLE 1: Comparison between this work and other published works.

\begin{tabular}{|c|c|c|c|c|c|}
\hline Ref. & Resonator & $f_{0}[\mathrm{GHz}]$ & FBW [\%] & Length $\left[\lambda_{g}\right]$ & IL $[\mathrm{dB}]$ \\
\hline [9] & SRR & 4.6 & 1.7 & 0.53 & 5.2 \\
\hline [8] & EBG & 5 & 3.7 & 0.47 & 2.8 \\
\hline [6] & CSRR & 1 & 8 & 0.42 & 1.5 \\
\hline [7] & Multisection SIR & 2.4 & 10.8 & 0.24 & 5.8 \\
\hline This work & Quasi-planar chiral & 1 & 2.2 & 0.17 & 5.1 \\
\hline
\end{tabular}

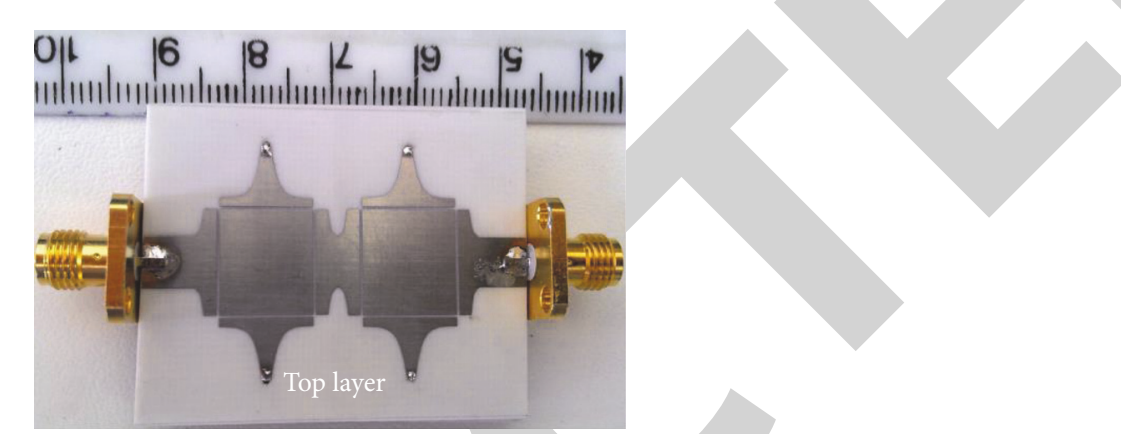

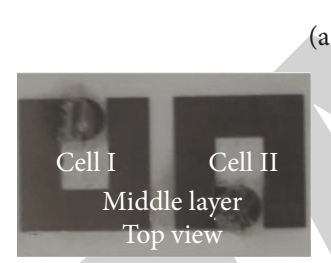

(b)

(a)

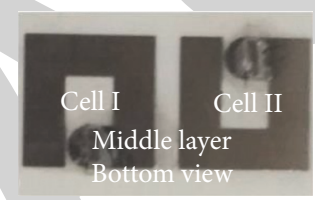

(c)

FIGURE 6: The layers of the fabricated filter: (a) top layer, (b) top view of the middle layer, and (c) bottom view of the middle layer (notice that the bottom layer (not shown in this figure) is ground plane).

in Table 1 (which is sorted by filter length in descending order) indicating that the proposed filter with $0.17 \lambda_{g}$ length is the most compact (the operating frequency of the proposed filter is the lowest, but its size is the smallest), yet with FBW = $2.2 \%$ the second narrowest. On the other hand, the insertion loss of the filter in this work is still acceptable.

\section{Conclusion}

In this article, a narrow bandwidth bandpass filter based on the quasi-planar chiral resonators is presented. The proposed filter has the merits of simple design, backward propagation, compact size, and high selectivity. The results of equivalent circuit simulation, full-wave simulation, and measurement are in good agreement. Compared with other similar filters, it could be observed that the proposed filter has the advantages of better size integration and frequency selectivity. The proposed filter is feasible and applicable in modern microwave communication circuits.

\section{Appendix}

The input impedance $\left(Z_{\text {in }}\right)$ indicated in Figure $5(\mathrm{~b})$ at the resonant frequency of the quasi-planar resonator $\omega_{0, p}$ (where
$\left.Z_{p}=\infty\right)$ can be written as $Z_{\text {in }}=\left[\left(Z_{0}+Z_{s}\right) / / Z_{\text {stub }} / 2+Z_{s}\right]$. Hence, $Z_{\text {stub }}$ at $\omega_{0, p}$ is derived as

$$
\begin{aligned}
& Z_{\text {stub }}\left(\omega_{0, p}\right) \\
& \quad=2 \frac{\left[Z_{0}+Z_{s}\left(\omega_{0, p}\right)\right]\left[Z_{\text {in }}\left(\omega_{0, p}\right)-Z_{s}\left(\omega_{0, p}\right)\right]}{Z_{0}+2 Z_{s}\left(\omega_{0, p}\right)-Z_{\text {in }}\left(\omega_{0, p}\right)} .
\end{aligned}
$$

$Z_{\text {stub }}$ is a complex amount whose real part describes losses which is ignored here for simplicity. Considering Figure 5(b), it can be obtained that

$$
\mathfrak{I}\left(Z_{\text {stub }}\right)=j \omega L_{\text {stub }}-\frac{j}{\omega C_{\text {stub }}} .
$$

And the resonant frequency of $L C$ network is

$$
\omega_{0, s}=\frac{1}{\sqrt{L_{\text {stub }} C_{\text {stub }}}} .
$$

Then, $L_{\text {stub }}$ and $C_{\text {stub }}$ can be extracted as

$$
\begin{aligned}
L_{\text {stub }} & =\frac{\omega_{0, p} \mathfrak{I}\left(Z_{\text {stub }}\left(\omega_{0, p}\right)\right)}{\Delta \omega_{0}^{2}} \\
C_{\text {stub }} & =\frac{\Delta \omega_{0}^{2}}{\omega_{0, p} \omega_{0, s}^{2} \mathfrak{I}\left(Z_{\text {stub }}\left(\omega_{0, p}\right)\right)},
\end{aligned}
$$




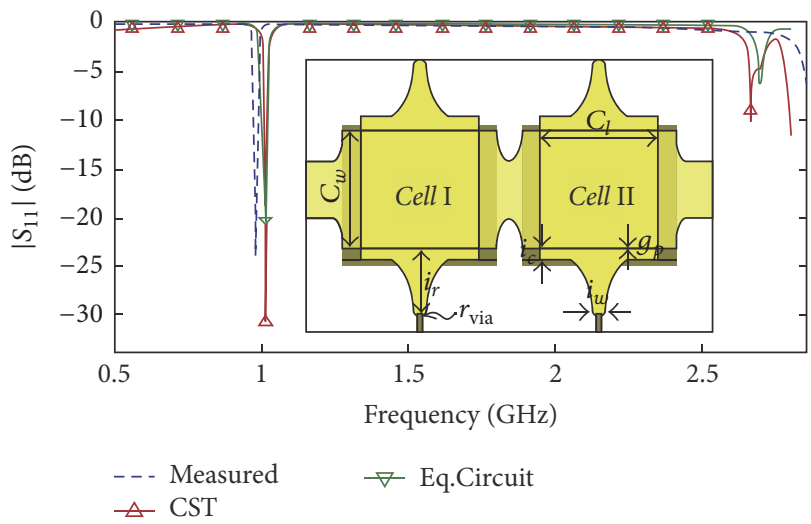

(a)

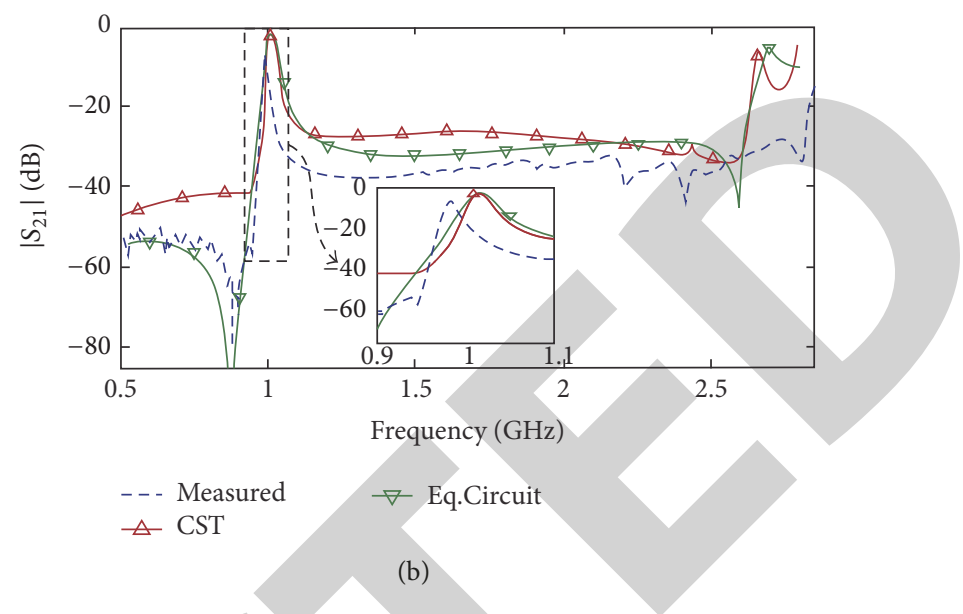

Figure 7: Measured, full-wave, and equivalent circuit simulations of (a) $\left|S_{11}\right|$ and (b) $\left|S_{21}\right|$. Layout parameters are $i_{r}=6.5 \mathrm{~mm}, i_{c}=1 \mathrm{~mm}, i_{w}$ $=1 \mathrm{~mm}, r_{\text {via }}=0.25 \mathrm{~mm}$, and $g_{p}=0.2 \mathrm{~mm}$. Extracted values of circuit model elements are $L_{\text {stub }}=10.8 \mathrm{nH}$ and $C_{\text {stub }}=0.35 \mathrm{pF}$.

where

$$
\Delta \omega_{0}^{2}=\omega_{0, p}^{2}-\omega_{0, s}^{2}
$$

\section{Competing Interests}

The authors declare that there is no conflict of interests regarding the publication of this paper.

\section{Acknowledgments}

The work is supported by National Natural Science Foundation of China (no. 61563015), Young Foundation of Humanities and Social Sciences of Ministry of Education in China (no. 13YJCZH089), and Young Foundation of Educational Commission of Jiangxi Province of China (no. GJJ14401).

\section{References}

[1] R. Markques, F. Martin, and M. Sorolla, Metamaterials with Negative Parameters: Theory, Design and Microwave Applications, Wiley Interscience, 2008.

[2] F. Aznar, J. García-García, M. Gil, J. Bonache, and F. Martín, "Strategies for the miniaturization of metamaterial resonators," Microwave and Optical Technology Letters, vol. 50, no. 5, pp. 1263-1270, 2008.

[3] R. Marqués, L. Jelinek, and F. Mesa, "Negative refraction from balanced quasi-planar chiral inclusions," Microwave and Optical Technology Letters, vol. 49, no. 10, pp. 2606-2609, 2007.

[4] S. M. Hashemi, M. Soleimani, and S. A. Tretyakov, "Compact negative-epsilon stop-band structures based on double-layer chiral inclusions," IET Microwaves, Antennas and Propagation, vol. 7, no. 8, pp. 621-629, 2013.

[5] S.-G. Mao, M.-S. Wu, Y.-Z. Chueh, and C. H. Chen, "Modeling of symmetric composite right/left-handed coplanar waveguides with applications to compact bandpass filters," IEEE Transactions on Microwave Theory and Techniques, vol. 53, no. 11, pp. 3460-3466, 2005.
[6] J. Bonache, I. Gil, J. García-García, and F. Martín, "Novel microstrip bandpass filters based on complementary splitring resonators," IEEE Transactions on Microwave Theory and Techniques, vol. 54, no. 1, pp. 265-271, 2006.

[7] H. Zhang and K. J. Chen, "Miniaturized coplanar waveguide bandpass filters using multisection stepped-impedance resonators," IEEE Transactions on Microwave Theory and Techniques, vol. 54, no. 3, pp. 1090-1095, 2006.

[8] S.-G. Mao and Y.-Z. Chueh, "Coplanar waveguide bandpass filters with compact size and wide spurious-free stopband using electromagnetic bandgap resonators," IEEE Microwave and Wireless Components Letters, vol. 17, no. 3, pp. 181-183, 2007.

[9] A. L. Borja, J. Carbonell, V. E. Boria, J. Cascon, and D. Lippens, "A $2 \%$ bandwidth C-band filter using cascaded split ring resonators," IEEE Antennas and Wireless Propagation Letters, vol. 9, pp. 256-259, 2010.

[10] V. G. Veselago, "The electrodynamics of substances with simultaneously negative values of $\varepsilon$ and $\mu$," Soviet Physics Uspekhi, vol. 10, no. 4, pp. 509-514, 1968.

[11] M. Studniberg and G. V. Eleftheriades, "A quad-band bandpass filter using negative-refractive-index transmission-line (NRITL) metamaterials," in Proceedings of the IEEE Antennas and Propagation Society International Symposium (AP-S '07), pp. 4961-4964, Honolulu, Hawaii, USA, June 2007.

[12] Z.-P. Wang, Z.-Y. Han, and L.-M. Guo, "Miniaturized zerothorder resonator based on simplified CRLH TL structure," Microwave and Optical Technology Letters, vol. 53, no. 4, pp. 848-852, 2011.

[13] X. Q. Lin, Q. Cheng, R. P. Liu, D. Bao, and T. J. Cui, "Compact resonator filters and power dividers designed with simplified meta-structures," Journal of Electromagnetic Waves and Applications, vol. 21, no. 12, pp. 1663-1672, 2007.

[14] S. M. Jiang, W. T. Li, X. H. Wang, Q. Y. Song, and X. W. Shi, "A novel method of designing cross-coupled filters through optimization," Journal of Electromagnetic Waves and Applications, vol. 23, no. 14-15, pp. 2011-2019, 2009.

[15] N. Dolatsha, M. Shahabadi, and R. Dehbashi, "Via-free CPWbased composite right/left-handed transmission line and a calibration approach to determine its propagation constant," Journal of Electromagnetic Waves and Applications, vol. 22, no. 11-12, pp. 1599-1606, 2008. 
[16] H.-X. Xu, G.-M. Wang, Q. Peng, and J.-G. Liang, "Novel design of tri-band bandpass filter based on fractal-shaped geometry of a complementary single split ring resonator," International Journal of Electronics, vol. 98, no. 5, pp. 647-654, 2011.

[17] J.-Q. Gong, C.-H. Liang, and B. Wu, "Novel dual-band hybrid coupler using improved simplified CRLH transmission line stubs," Microwave and Optical Technology Letters, vol. 52, no. 11, pp. 2473-2476, 2010.

[18] Y. Guo and R. Xu, "Ultra-wideband power splitting/combining technique using zero-degree left handed transmission lines," Journal of Electromagnetic Waves and Applications, vol. 21, no. 8, pp. 1109-1118, 2007.

[19] J.-Y. Lee, D.-J. Kim, and J.-H. Lee, "High order bandpass filter using the first negative resonant mode of composite right/lefthanded transmission line," Microwave and Optical Technology Letters, vol. 51, no. 5, pp. 1182-1185, 2009.

[20] C.-H. Tseng and C.-L. Chang, "An image reject mixer with composite right/left-handed quadrature power splitter and if hybrid," Journal of Electromagnetic Waves and Applications, vol. 22, no. 11-12, pp. 1557-1564, 2008.

[21] J. He, B.-Z. Wang, and K.-H. Zhang, "Arbitrary dual-band coupler using accurate model of composite right/left handed transmission line," Journal of Electromagnetic Waves and Applications, vol. 22, no. 8-9, pp. 1267-1272, 2008.

[22] J.-S. Li and Y.-Y. Zhuang, "Compact microstrip bandpass filter using composite right/left-handed transmission lines," Microwave and Optical Technology Letters, vol. 49, no. 8, pp. 1929-1931, 2007.

[23] J. Ju and S. Kahng, "A compact UWB bandpass filter using a center-tapped composite right/left-handed transmission-line zeroth-order resonator," Microwave and Optical Technology Letters, vol. 53, no. 9, pp. 1974-1976, 2011.

[24] Q. Zhang and S. N. Khan, "Compact broadside coupled directional coupler based on coplanar CRLH waveguides," Journal of Electromagnetic Waves and Applications, vol. 23, no. 2-3, pp. 267-277, 2009.

[25] J. He, B.-Z. Wang, and K.-H. Zhang, "Wideband differential phase shifter using modified composite right/left handed transmission line," Journal of Electromagnetic Waves and Applications, vol. 22, no. 10, pp. 1389-1394, 2008.

[26] S.-W. Zhao, Z.-X. Tang, W. Dai, and B. Zhang, "A novel dual-layer high-directivity directional coupler using printed metamaterial MS/NRI coupled-line," Microwave and Optical Technology Letters, vol. 52, no. 12, pp. 2706-2708, 2010. 